\title{
Pair Sum Labeling of some Special Graphs
}

\author{
K.Manimekalai \\ Department of Mathematics, \\ Bharathi Women's College (Autonomous), \\ Chennai -600106, India.
}

\author{
K.Thirusangu \\ Department of Mathematics, \\ S.I.V.E.T.College, Gowrivakkam, \\ Chennai -600073, India.
}

\begin{abstract}
Let $\mathrm{G}$ be a (p, q) graph. A one-one map $\mathrm{f}: \mathrm{V}(\mathrm{G}) \rightarrow\{ \pm 1, \pm 2$, $\ldots, \pm p\}$ is said to be a pair sum labeling if the induced edge function, $\mathrm{f}_{\mathrm{e}}: \mathrm{E}(\mathrm{G}) \rightarrow \mathrm{Z}-\{0\}$ defined by $\mathrm{f}_{\mathrm{e}}(\mathrm{uv})=\mathrm{f}(\mathrm{u})+\mathrm{f}(\mathrm{v})$ is one-one and $\mathrm{f}_{\mathrm{e}}(\mathrm{E}(\mathrm{G}))$ is either of the form $\left\{ \pm \mathrm{k}_{1}, \pm \mathrm{k}_{2}, \ldots, \pm \mathrm{k}_{\mathrm{q} / 2}\right\}$ or $\left\{ \pm \mathrm{k}_{1}, \pm \mathrm{k}_{2}, \ldots, \pm \mathrm{k}_{(\mathrm{q}-1) / 2}\right\} \cup\left\{\mathrm{k}_{(\mathrm{q}+1) / 2}\right\}$ according as $\mathrm{q}$ is even or odd. Recently, the pair sum labeling was introduced by R.Ponraj, J. V. X. Parthipan [3]. In this paper we study about the pair sum labeling of the coconut tree $\mathrm{CT}(\mathrm{m}, \mathrm{n})$, the Y-tree $\mathrm{Y}_{\mathrm{n}+1}$, the Jelly fish graph $\mathrm{J}(\mathrm{m}, \mathrm{n})$, the $(\mathrm{m}, 2)$-kite, $(\mathrm{m}, 1)$-kite, the theta graph $\Theta\left(l^{[m]}\right)$, for $m$ even and complete binary tree.
\end{abstract}

\section{Keywords}

pair sum labeling, pair sum graph.

\section{AMS Classification: $05 \mathrm{C} 78$.}

\section{INTRODUCTION}

The graph considered here are all finite, undirected and simple. $V(G)$ and $E(G)$ denote the vertex set and edge set of a graph G. The pair sum labeling is introduced in [3] by $R$. Ponraj and et al. In [3],[4] [5] and [6] they study the pair sum labeling of cycle, path, star and some of their related graphs like $B_{m, n}, K_{1, n} \cup K_{1, m}, P_{m} \cup K_{1, n}, C_{n} \cup C_{n}$ etc. In this paper we prove that the pair sum labeling of coconut tree $\mathrm{CT}(\mathrm{m}, \mathrm{n})$, the Y-tree $\mathrm{Y}_{\mathrm{n}+1}$, Jelly fish $\mathrm{J}(\mathrm{m}, \mathrm{n}),(\mathrm{m}, 2)$-kite, $(\mathrm{m}, 1)$-kite and the theta graph $\Theta\left(l^{[m]}\right)$, for $m$ even and complete binary tree. Let $\mathrm{x}$ be any real number, then $[\mathrm{x}]$ denotes the largest integer less than or equal to $\mathrm{x}$. Terms and terminology as in Harary [2].

Definition 1.1[3]: Let G(V, E) be a (p, q) graph. A oneone function $\mathrm{f}: \mathrm{V}(\mathrm{G}) \rightarrow\{ \pm 1, \pm 2, \ldots, \pm \mathrm{p}\}$ is said to be a pair sum labeling if the induced edge function $\mathrm{f}_{\mathrm{e}}: \mathrm{E}(\mathrm{G}) \rightarrow \mathrm{Z}-\{0\}$ defined by $f_{e}(u v)=f(u)+f(v)$ is one-one and $f_{e}(E(G))$ is either of the form $\left\{ \pm \mathrm{k}_{1}, \pm \mathrm{k}_{2}, \ldots, \pm \mathrm{k}_{\mathrm{q} / 2}\right\}$ or $\left\{ \pm \mathrm{k}_{1}, \pm \mathrm{k}_{2}, \ldots, \pm \mathrm{k}_{(\mathrm{q}-1) / 2}\right\} \cup$ $\left\{\mathrm{k}_{(\mathrm{q}+1) / 2}\right\}$ according as $\mathrm{q}$ is even or odd. A graph with a pair sum labeling defined on it is called a pair sum graph.

Definition 1.2: A coconut Tree $\mathrm{CT}(\mathrm{m}, \mathrm{n})$ is the graph obtained from the path $\mathrm{P}_{\mathrm{m}}$ by appending $\mathrm{n}$ new pendent edges at an end vertex of $\mathrm{P}_{\mathrm{m}}$.

Definition 1.3: A Y -tree $Y_{n+1}$ is a graph obtained from the path $P_{n}$ by appending an edge to a vertex of the path $P_{m}$ adjacent to an end point.

Definition 1.4: An $(n, t)$-kite is a cycle of length $n$ with a t-edge path (the tail) attached to one vertex. In particular, the $(\mathrm{n}, 1)$-kite is a cycle of length $\mathrm{n}$ with an edge attached to one vertex. $(n, 1)$-kite is also known as flag $F \ell_{n}$.
Definition 1.5: The Jelly fish graph $J(m, n)$ is obtained from a 4- cycle $v_{1}, v_{2}, v_{3}, v_{4}$ by joining $v_{1}$ and $v_{3}$ with an edge and appending $\mathrm{m}$ pendent edges to $\mathrm{v}_{2}$ and $\mathrm{n}$ pendent edges to $\mathrm{v}_{4}$.

Definition 1.6: Take $\mathrm{k}$ paths of length $l_{l}, l_{2}, l_{3} \ldots, l_{k}$, where $\mathrm{k} \geq 3$ and $l_{i}=1$ for at most one i. Identify their end points to form a new graph. The new graph is called a generalized theta graph, and is denoted by $\Theta\left(l_{1}, l_{2}, l_{3, \ldots}, l_{k}\right)$. In other words, $\Theta\left(l_{1} l_{2}, l_{3} \ldots l_{k}\right)$ consists $\mathrm{k} \geq 3$ pair wise internally disjoint paths of length $l_{1}, l_{2}, l_{3} \ldots, l_{k}$ that share a pair of common end points $\mathrm{u}$ and $\mathrm{v}$. If each $l_{i}(i=1,2, \ldots, k)$ is equal to 1 , we will write $\Theta\left(l^{[\mathrm{k}]}\right)$.

Definition 1.7: A binary tree is defined as a tree in which there is exactly one vertex (root vertex) of degree two and each of the remaining vertices is of degree one or three. A d- level complete binary tree (complete binary tree of depth d) is a binary tree in which each internal vertex (non-pendent vertex) other than the root is of degree three and all the pendent vertices are at level $\mathrm{d}$. ( In a binary tree a vertex $\mathrm{v}$ is said to be at level $l$, if $\mathrm{v}$ is at a distance of $l$ from the root).

In [3] and [2] R. Ponraj, J. V. X. Parthipan have proved the following results which we will use in the proof of our theorems.

Theorem 1.1[3]: $(3,1)$ - kite is not a pair sum graph. Theorem 1.2[2]: The complete bipartite graphs $K_{1, \mathrm{n}}$ and $\mathrm{K}_{2, \mathrm{n}}$ are pair sum graphs.

\section{MAIN RESULTS}

Theorem 2.1: The (m, 2)-kite is a pair sum graph for all $\mathrm{m} \geq 3$.

Proof: Let V be the vertex set and $\mathrm{E}$ be the edge set of ( $\mathrm{m}$, 2)-kite. Then $|\mathrm{V}|=|\mathrm{E}|=\mathrm{m}+2$. Consider the following two cases.

Case 1: $\mathrm{m}$ is even. Let $\mathrm{m}=2 \mathrm{r}$.

Let $\mathrm{V}=\left\{\mathrm{u}_{\mathrm{i}}, \mathrm{v}_{\mathrm{i}} / 1 \leq \mathrm{i} \leq \mathrm{r}\right\} \cup\left\{\mathrm{w}_{1}, \mathrm{w}_{2}\right\}$ and

$\mathrm{E}=\left\{\mathrm{u}_{\mathrm{i}} \mathrm{u}_{\mathrm{i}+1}, \mathrm{v}_{\mathrm{i}} \mathrm{v}_{\mathrm{i}+1} / 1 \leq \mathrm{i} \leq \mathrm{r}-1\right\} \cup\left\{\mathrm{u}_{\mathrm{r}} \mathrm{v}_{1}, \mathrm{v}_{\mathrm{r}} \mathrm{u}_{1}, \mathrm{u}_{\mathrm{r}} \mathrm{w}_{1}, \mathrm{w}_{1} \mathrm{w}_{2}\right\}$. Define a map $\mathrm{f}: \mathrm{V} \rightarrow\{ \pm 1, \pm 2, \ldots, \pm(2 \mathrm{r}+2)\}$ as follows:

$\mathrm{f}\left(\mathrm{u}_{\mathrm{i}}\right)=2 \mathrm{i} ; 1 \leq \mathrm{i} \leq \mathrm{r}-1$

$\mathrm{f}\left(\mathrm{v}_{\mathrm{i}}\right)=-\mathrm{f}\left(\mathrm{u}_{\mathrm{i}}\right) ; 1 \leq \mathrm{i} \leq \mathrm{r}-1$

$\mathrm{f}\left(\mathrm{u}_{\mathrm{r}}\right)=1, \mathrm{f}\left(\mathrm{v}_{\mathrm{r}}\right)=-1, \mathrm{f}\left(\mathrm{w}_{1}\right)=-(\mathrm{r}+1), \mathrm{f}\left(\mathrm{w}_{2}\right)=(2 \mathrm{r}+1)$.

Then $\mathrm{f}$ is a pair sum labeling.

Case 2: $\mathrm{m}$ is odd.

Subcase 2.1: $\mathrm{m} \geq 9$

Let $\mathrm{m}=2 \mathrm{r}+1, \mathrm{r} \geq 4$.

Let $\mathrm{V}=\left\{\mathrm{u}_{\mathrm{i}} / 1 \leq \mathrm{i} \leq \mathrm{r}\right\} \cup\left\{\mathrm{v}_{\mathrm{i}} / 1 \leq \mathrm{i} \leq \mathrm{r}+1\right\} \cup\left\{\mathrm{w}_{1}, \mathrm{w}_{2}\right\}$ and $\mathrm{E}=\left\{\mathrm{u}_{\mathrm{i}} \mathrm{u}_{\mathrm{i}+1} / 1 \leq \mathrm{i} \leq \mathrm{r}-1\right\} \cup\left\{\mathrm{v}_{\mathrm{i}} \mathrm{v}_{\mathrm{i}+1} / 1 \leq \mathrm{i} \leq \mathrm{r}\right\} \cup\left\{\mathrm{u}_{\mathrm{r}} \mathrm{v}_{1}, \mathrm{v}_{\mathrm{r}+1} \mathrm{u}_{1}\right.$, $\left.\mathrm{v}_{\mathrm{r}} \mathrm{w}_{1}, \mathrm{w}_{1} \mathrm{w}_{2}\right\}$. 
Define $\mathrm{f}: \mathrm{V} \rightarrow\{ \pm 1, \pm 2, \ldots, \pm(2 \mathrm{r}+3)\}$ as follows:

$\mathrm{f}\left(\mathrm{u}_{\mathrm{i}}\right)=2 \mathrm{i} ; 1 \leq \mathrm{i} \leq \mathrm{r}-1$

$\mathrm{f}\left(\mathrm{v}_{\mathrm{i}}\right)=-\mathrm{f}\left(\mathrm{u}_{\mathrm{i}}\right) ; 1 \leq \mathrm{i} \leq \mathrm{r}-2$

$\mathrm{f}\left(\mathrm{u}_{\mathrm{r}}\right)=2 \mathrm{r}-1, \mathrm{f}\left(\mathrm{v}_{\mathrm{r}-1}\right)=2 \mathrm{r}-3, \mathrm{f}\left(\mathrm{v}_{\mathrm{r}}\right)=-(2 \mathrm{r}-2), \mathrm{f}\left(\mathrm{v}_{\mathrm{r}+1}\right)=-(2 \mathrm{r}-1)$

$\mathrm{f}\left(\mathrm{w}_{1}\right)=-(2 \mathrm{r}-5), \mathrm{f}\left(\mathrm{w}_{2}\right)=2 \mathrm{r}+3$.

Then $\mathrm{f}$ is a pair sum labeling.

Subcase 2.2: for $\mathrm{m}=3,5$ and 7 , a pair sum labeling of $(\mathrm{m}, 2)$-kite is given in Figure 1.
Subcase 1.1: $\mathrm{m}$ is even. Let $\mathrm{m}=2 \mathrm{r}$. Then $\mathrm{V}(\mathrm{G})=\left\{\mathrm{u}_{\mathrm{i}}, \mathrm{v}_{\mathrm{i}} / 1\right.$ $\leq \mathrm{i} \leq \mathrm{r}\} \cup\left\{\mathrm{w}_{\mathrm{i}} / 1 \leq \mathrm{i} \leq \mathrm{n}\right\}$ and

$\mathrm{E}(\mathrm{G})=\left\{\mathrm{u}_{\mathrm{r}} \mathrm{v}_{1}, \mathrm{u}_{\mathrm{i}} \mathrm{u}_{\mathrm{i}+1}, \mathrm{v}_{\mathrm{i}} \mathrm{v}_{\mathrm{i}+1} / 1 \leq \mathrm{i} \leq \mathrm{r}-1\right\} \cup\left\{\mathrm{w}_{\mathrm{i}} / 1 \leq \mathrm{i} \leq \mathrm{n}\right\}$.

Define $\mathrm{f}: \mathrm{V} \rightarrow\{ \pm 1, \pm 2, \ldots, \pm(2 \mathrm{r}+\mathrm{n})\}$ as follows:

$\mathrm{f}\left(\mathrm{u}_{1}\right)=-3, \mathrm{f}\left(\mathrm{u}_{\mathrm{r}}\right)=1, \mathrm{f}\left(\mathrm{v}_{\mathrm{r}}\right)=-1$

$\mathrm{f}\left(\mathrm{u}_{\mathrm{i}}\right)=2 \mathrm{i} ; 2 \leq \mathrm{i} \leq \mathrm{r}-1$

$\mathrm{f}\left(\mathrm{v}_{\mathrm{i}}\right)=-\mathrm{f}\left(\mathrm{u}_{\mathrm{i}}\right) ; 1 \leq \mathrm{i} \leq \mathrm{r}-1$

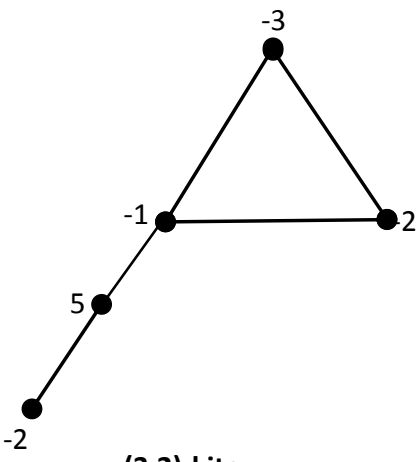

$(3,2)$-kite

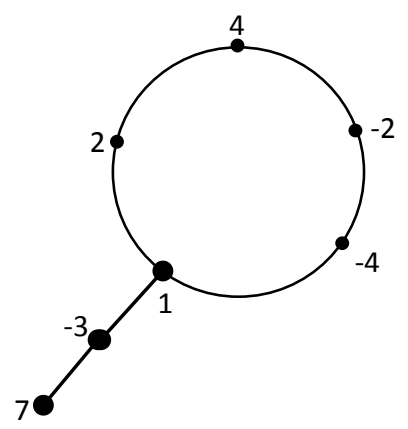

(5,2)-kite

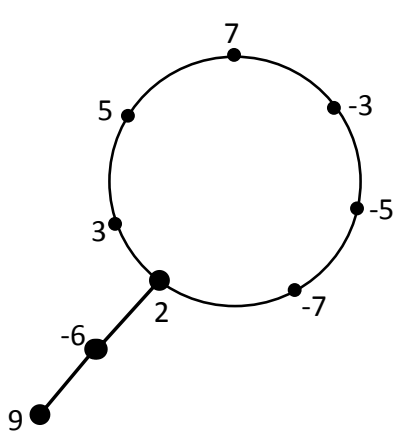

(7,2)-kite

Figure 1

Hence the $(\mathrm{m}, 2)$-kite is a pair sum graph for all $\mathrm{m} \geq 3$.

Corollary 2.2: By deleting the pendant vertex in the $(\mathrm{m}, 2)$-kite of the proof of the Theorem 2.1 and by Theorem 1.1 , the $(\mathrm{m}, 1)$-kite is a pair sum graph except for $\mathrm{m}=3$.

Example 2.1: In Figure 2, pair sum labeling of (16,2)kite and (17,2)-kite are illustrated.

$$
\mathrm{f}\left(\mathrm{w}_{\mathrm{i}}\right)= \begin{cases}7 & ; \quad \mathrm{i}=1 \\ -(2 \mathrm{i}+3) & ; \quad 2 \leq i \leq n \& \mathrm{i} \text { even } \\ (2 \mathrm{i}+3) & ; \quad 3 \leq i \leq n \& \mathrm{i} \text { odd }\end{cases}
$$

Subcase 1.2: $\mathrm{m}$ is odd. Let $\mathrm{m}=2 \mathrm{r}+1$.

Then $\mathrm{V}(\mathrm{G})=\left\{\mathrm{u}_{\mathrm{r}+1}, \mathrm{u}_{\mathrm{i}}, \mathrm{v}_{\mathrm{i}} / 1 \leq \mathrm{i} \leq \mathrm{r}\right\} \cup\left\{\mathrm{w}_{\mathrm{i}} / 1 \leq \mathrm{i} \leq \mathrm{n}\right\}$ and $\mathrm{E}(\mathrm{G})=\left\{\mathrm{u}_{\mathrm{i}} \mathrm{u}_{\mathrm{i}+1}, \mathrm{u}_{\mathrm{r}} \mathrm{v}_{1}, \mathrm{v}_{\mathrm{r}} \mathrm{v}_{\mathrm{r}+1}, \mathrm{v}_{\mathrm{i}} \mathrm{v}_{\mathrm{i}+1} / 1 \leq \mathrm{i} \leq \mathrm{r}-1\right\} \cup\left\{\mathrm{v}_{\mathrm{r}+1} \mathrm{w}_{\mathrm{i}} / 1 \leq\right.$ $\mathrm{i} \leq \mathrm{n}\}$.
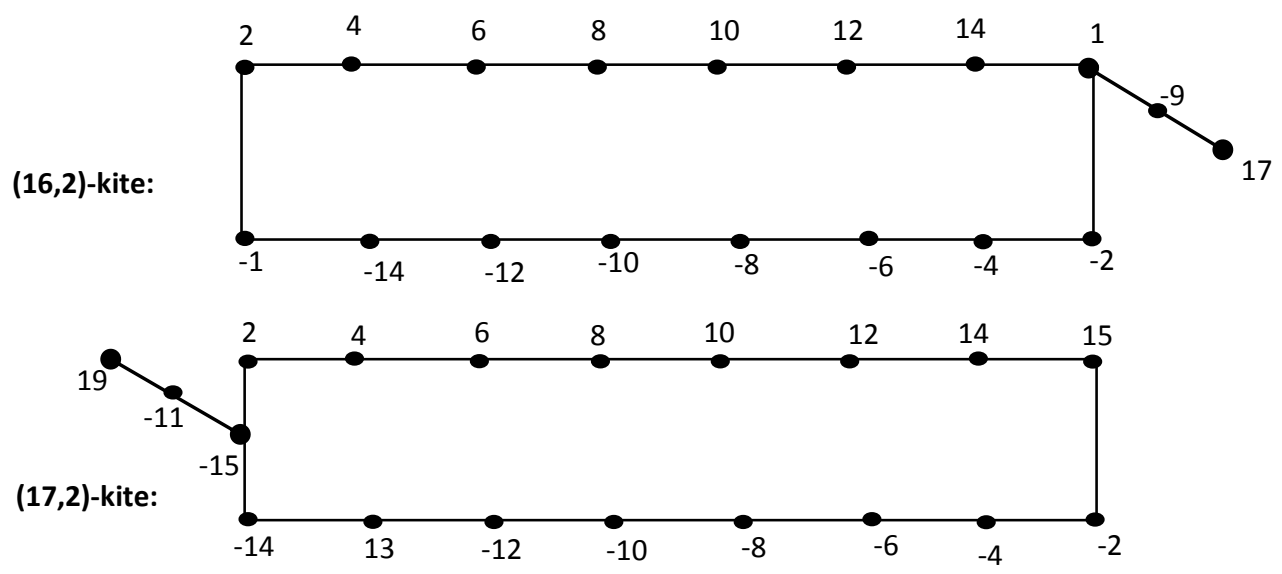

Figure 2: Pair sum labeling of $(16,2)$-kite and $(17,2)$-kite

Theorem 2.3: The coconut tree $\mathrm{CT}(\mathrm{m}, \mathrm{n})$ is a pair sum graph.

Proof: Let $\mathrm{G}(\mathrm{V}, \mathrm{E})=\mathrm{CT}(\mathrm{m}, \mathrm{n})$. then $|\mathrm{V}(\mathrm{G})|=\mathrm{m}+\mathrm{n}$ and $|\mathrm{E}(\mathrm{G})|=\mathrm{m}+\mathrm{n}-1$

Case 1: $\mathrm{m} \geq 5$
Define $f: V(G) \rightarrow\{ \pm 1, \pm 2, \ldots, \pm(2 r+n+1)\}$ as follows:

$$
\mathrm{f}\left(\mathrm{u}_{\mathrm{i}}\right)= \begin{cases}2(\mathrm{i}+1) & ; \quad 1 \leq \mathrm{i} \leq \mathrm{r}-1 \\ 1 & ; \mathrm{i}=\mathrm{r} .\end{cases}
$$




$$
\begin{aligned}
& \mathrm{f}\left(\mathrm{v}_{\mathrm{i}}\right)= \begin{cases}-2 \mathrm{i} & ; \quad 1 \leq \mathrm{i} \leq \mathrm{r} \\
-1 \quad ; \quad \mathrm{i}=\mathrm{r}+1\end{cases} \\
& \mathrm{f}\left(\mathrm{w}_{\mathrm{i}}\right)= \begin{cases}2 & ; \quad \mathrm{i}=1 \\
7 & ; \quad i=2 \\
-(2 \mathrm{i}+1) & ; \quad 3 \leq i \leq n\end{cases}
\end{aligned}
$$

Case 2: $\mathrm{m} \leq 4$.

Subcase 2.1: $\mathrm{m}=1$, then $\mathrm{G}=\mathrm{CT}(1, \mathrm{n})$ is the star $\mathrm{K}_{1, \mathrm{n}}$. By Theorem 1.2 [2], $\mathrm{G}$ is a pair sum graph.

Subcase 2.2: $\mathrm{m}=2$, then $\mathrm{G}=\mathrm{CT}(2, \mathrm{n})$ is the star $\mathrm{K}_{1, \mathrm{n}+1}$. By Theorem 1.2 [2], it is a pair sum graph.

Subcase 2.3: $\mathrm{m}=3$, then $\mathrm{G}=\mathrm{CT}(3, \mathrm{n})$.

Let $\mathrm{V}(\mathrm{G})=\left\{\mathrm{v}_{\mathrm{i}} / 1 \leq \mathrm{i} \leq 3\right\} \cup\left\{\mathrm{w}_{\mathrm{i}} / 1 \leq \mathrm{i} \leq \mathrm{n}\right\}$ and $\mathrm{E}(\mathrm{G})=\left\{\mathrm{v}_{1} \mathrm{v}_{2}\right.$, $\left.\mathrm{v}_{2} \mathrm{v}_{3}\right\} \cup\left\{\mathrm{v}_{3} \mathrm{w}_{\mathrm{i}} / 1 \leq \mathrm{i} \leq \mathrm{n}\right\}$.

Label the vertices $\mathrm{v}_{1}, \mathrm{v}_{2}$ and $\mathrm{v}_{3}$ by $-3,2$ and -1 respectively.

For $1 \leq \mathrm{i} \leq \mathrm{n}$, define $\mathrm{f}\left(\mathrm{w}_{\mathrm{i}}\right)= \begin{cases}-(i+1) & ; \text { i odd } \\ (i+2) & ; \text { i even }\end{cases}$

Subcase 2.4: $\mathrm{m}=4$ then $\mathrm{G}=\mathrm{CT}(4, \mathrm{n})$.

$\mathrm{V}(\mathrm{G})=\left\{\mathrm{v}_{\mathrm{i}} / 1 \leq \mathrm{i} \leq 4\right\} \cup\left\{\mathrm{w}_{\mathrm{i}} / 1 \leq \mathrm{i} \leq \mathrm{n}\right\}$ and $\mathrm{E}(\mathrm{G})=\left\{\mathrm{v}_{1} \mathrm{v}_{2}\right.$, $\left.\mathrm{v}_{2} \mathrm{v}_{3}, \mathrm{v}_{3} \mathrm{v}_{4}\right\} \cup\left\{\mathrm{v}_{4} \mathrm{w}_{\mathrm{i}} / 1 \leq \mathrm{i} \leq \mathrm{n}\right\}$. Label the vertices $\mathrm{v}_{1}, \mathrm{v}_{2}, \mathrm{v}_{3}$ and $\mathrm{v}_{4}$ by $-2,-4,1$ and 2 respectively.

For $1 \leq \mathrm{i} \leq \mathrm{n}$, define

$$
\mathrm{f}\left(\mathrm{w}_{\mathrm{i}}\right)= \begin{cases}4 & ; \mathrm{i}=1 \\ -1 & ; \mathrm{i}=2 \\ -3 & ; \mathrm{i}=3 \\ \mathrm{i}-1 & ; 4 \leq i \leq n \& \mathrm{i} \text { even } \\ -(\mathrm{i}+2) & ; 5 \leq i \leq n \& \mathrm{i} \text { odd }\end{cases}
$$

Then $\mathrm{f}$ is a pair sum labeling.

Hence the coconut tree $\mathrm{CT}(\mathrm{m}, \mathrm{n})$ is a pair sum graph.

Example 2.2: In Figure 3, the pair sum labeling for $\mathrm{CT}(4,7)$ is exhibited.

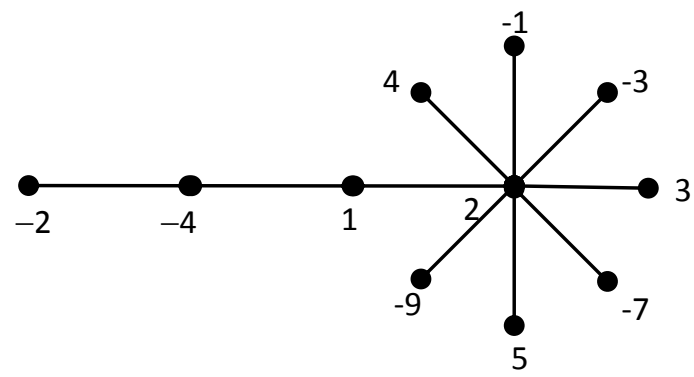

Figure. 3: Pair sum labeling $\mathrm{CT}(4,7)$

Corollary 2. 4: For $r \geq 3$, the $Y$-tree $Y_{r+1}$ is a pair sum graph.

Proof: It is easily observed that $Y_{r+1} \cong C T(r-1,2)$ for $r \geq 3$. Hence by Theorem 2.1, $\mathrm{Y}_{\mathrm{r}+1}$ is a pair sum graph.

Example 2.3: In Figure 4 a pair sum labeling of $Y_{4+1}$ and $\mathrm{Y}_{5+1}$ are illustrated.

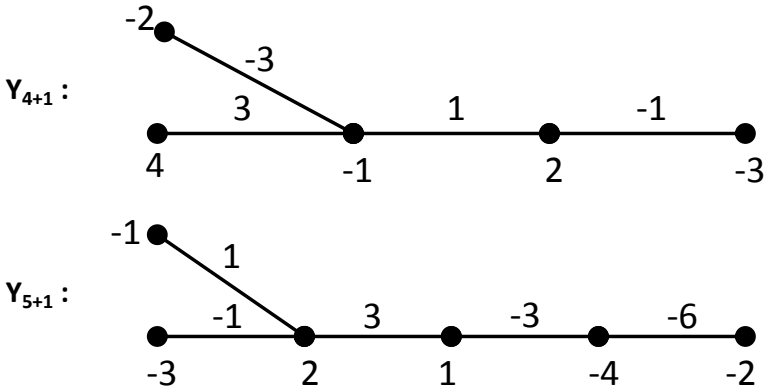

Figure 4: pair sum labeling of $Y_{6+1}, Y_{4+1}$ and $Y_{5+1}$

Theorem 2.5: For $m, n \geq 1$, Jelly fish graph $J(m, n)$ is a pair sum graph.

Proof: Let $G(V, E)=J(m, n)$. Then $G$ has $(m+n+4)$ vertices and $(m+n+5)$ edges. Let $n \geq m$. Let $V(G)=V_{1} \cup V_{2}$ where $\mathrm{V}_{1}=\{\mathrm{x}, \mathrm{u}, \mathrm{y}, \mathrm{v}\}, \mathrm{V}_{2}=\left\{\mathrm{u}_{\mathrm{i}}, \mathrm{v}_{\mathrm{j}} ; 1 \leq \mathrm{i} \leq \mathrm{m}, 1 \leq \mathrm{i} \leq \mathrm{n}\right\}$ and $\mathrm{E}=$ $\mathrm{E}_{1} \cup \mathrm{E}_{2}$, where $\mathrm{E}_{1}=\{\mathrm{xu}, \mathrm{uy}, \mathrm{yv}, \mathrm{vx}, \mathrm{xy}\}, \mathrm{E}_{2}=\left\{\mathrm{uu}_{\mathrm{i}}, \mathrm{vv}_{\mathrm{j}} ; 1 \leq\right.$ $\mathrm{i} \leq \mathrm{m}, 1 \leq \mathrm{j} \leq \mathrm{n}\}$.

Define $\mathrm{f:} \mathrm{V} \rightarrow\{ \pm 1, \pm 2, \ldots, \pm(\mathrm{m}+\mathrm{n}+4)\}$ as follows:

Label the vertices $\mathrm{x}, \mathrm{u}, \mathrm{y}, \mathrm{v}$ by $-5,-1,3$ and 1 respectively. Define

$$
\mathrm{f}\left(\mathrm{v}_{\mathrm{i}}\right)=\left\{\begin{array}{lll}
5 & ; & \mathrm{i}=1 \\
2(\mathrm{i}-1) & ; & 2 \leq i \leq 4 \\
(\mathrm{i}+2) & ; & 5 \leq i \leq m+1
\end{array}\right.
$$

For $2 \leq \mathrm{j} \leq \mathrm{n}-\mathrm{m}$

$\mathrm{f}\left(\mathrm{v}_{\mathrm{m}+\mathrm{j}}\right)=\left\{\begin{array}{lll}m+3+(j / 2) & ; & 2 \leq j \leq m-n \& \text { jeven } \\ -(m+5+(j-1) / 2) & ; & 3 \leq j \leq m-n \& \mathrm{jodd}\end{array}\right.$ $\mathrm{f}\left(\mathrm{u}_{\mathrm{i}}\right)=-\mathrm{f}\left(\mathrm{v}_{\mathrm{i}+1}\right) ; 1 \leq \mathrm{i} \leq \mathrm{m}$.

Then $\mathrm{f}$ is a pair sum labeling. Hence the Jelly fish graph $\mathrm{J}(\mathrm{m}$, n) is a pair sum graph.

Example 2.4: In Figure 5, the pair sum labeling for the Jelly fish graph $\mathrm{J}(4,7)$ is exhibited.

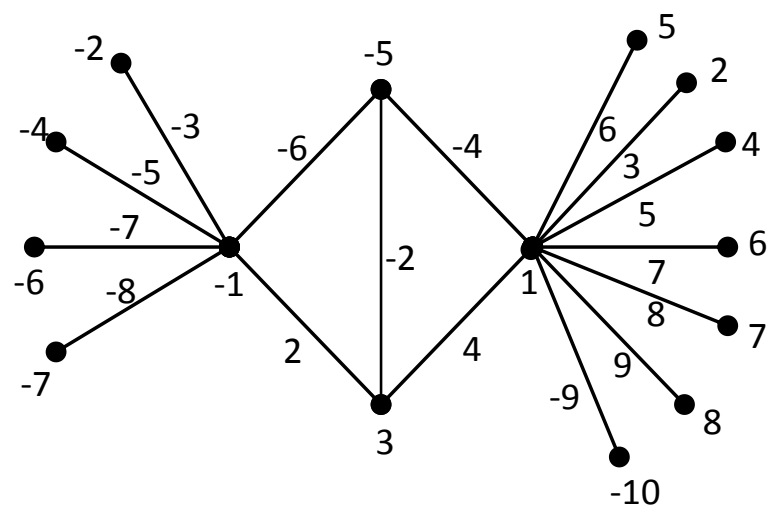

Figure 5: pair sum labeling for the Jelly fish $J(4,7)$

Next we will prove that the theta graph $\Theta\left(\ell^{[\mathrm{m}]}\right)$ is a pair sum graph, for m even.

Theorem 2.6: For $m$ even, the theta graph $\Theta\left(\ell^{[\mathrm{m}]}\right)$ is a pair sum graph. 
Proof: Let $\mathrm{G}(\mathrm{V}, \mathrm{E})=\Theta\left(\ell^{[\mathrm{m}]}\right)$. Then $|\mathrm{V}(\mathrm{G})|=\mathrm{m}(\ell-1)+2$ and $|\mathrm{E}(\mathrm{G})|=\mathrm{m} \ell$. Let $\mathrm{V}(\mathrm{G})=\mathrm{V}_{1} \cup \mathrm{V}_{2}$ where $\mathrm{V}_{1}=\left\{\mathrm{u}_{\mathrm{i}, \mathrm{j}}, \mathrm{v}_{\mathrm{i}, \mathrm{j}} \mid 1 \leq \mathrm{i} \leq m / 2\right.$ and $\left.1 \leq \mathrm{j} \leq \ell-1\right\}$, $\mathrm{V}_{2}=\{\mathrm{u}, \mathrm{v}\}$ and $\mathrm{E}(\mathrm{G})=\mathrm{E}_{1} \cup \mathrm{E}_{2}$ where $\mathrm{E}_{1}=\left\{\mathrm{u}_{\mathrm{i}, \mathrm{j}} \mathrm{u}_{\mathrm{i}, \mathrm{j}+1}, \mathrm{v}_{\mathrm{i}, \mathrm{j}} \mathrm{v}_{\mathrm{i}, \mathrm{j}+1} / 1 \leq \mathrm{i} \leq m / 2,1 \leq \mathrm{j} \leq \ell-2\right\}$, $\mathrm{E}_{2}=\left\{\mathrm{uu}_{\mathrm{i}, 1}, \mathrm{u} \mathrm{v}_{\mathrm{i}, \ell-1}, \mathrm{vv}_{\mathrm{i}, 1}, \mathrm{v} \mathrm{u}_{\mathrm{i}, \ell-1} / 1 \leq \mathrm{i} \leq m / 2\right\}$.

Define a map $\mathrm{f}: \mathrm{V}(\mathrm{G}) \rightarrow\{ \pm 1, \pm 2, \ldots, \pm \mathrm{m}(\ell-1)+2\}$ as follows:

$\mathrm{f}(\mathrm{u})=1, \mathrm{f}(\mathrm{v})=-1$,

$\mathrm{f}\left(\mathrm{u}_{\mathrm{i}, \mathrm{j}}\right)=2(\ell \mathrm{i}-\mathrm{i}+\mathrm{j}-\ell+1) ; 1 \leq \mathrm{i} \leq \mathrm{m} / 2 \& 1 \leq \mathrm{j} \leq \ell-1$

$\left.\mathrm{f}\left(\mathrm{v}_{\mathrm{i}, \mathrm{j}}\right)=\mathrm{f}\left(\mathrm{u}_{\mathrm{i} . \mathrm{j}}\right)\right) ; 1 \leq \mathrm{i} \leq \mathrm{m} / 2 \& 1 \leq \mathrm{j} \leq \ell-1$

Clearly $\mathrm{f}$ is a pair sum labeling. Hence the theta graph $\Theta\left(\ell^{[\mathrm{m}]}\right)$ is a pair sum graph for m even.
Proof: Construct the m-level complete binary tree as shown in figure 2.6. Let the vertex set $\mathrm{V}$ and the edge set $\mathrm{E}$ be defined as $\mathrm{V}=\left\{\mathrm{x}_{0}\right\} \cup\left\{\mathrm{x}_{\mathrm{i}, \mathrm{j}} / 1 \leq \mathrm{i} \leq \mathrm{m}, 1 \leq \mathrm{j} \leq 2^{\mathrm{i}}\right\}$ and $\mathrm{E}=$ $\left\{\mathrm{x}_{0} \mathrm{x}_{1,1}, \mathrm{x}_{0} \mathrm{x}_{1,2}\right\} \cup\left\{\mathrm{x}_{\mathrm{i}, \mathrm{j}} \mathrm{x}_{\mathrm{i}+1,2 \mathrm{j}-1}, \mathrm{x}_{\mathrm{i}, \mathrm{j}} \mathrm{x}_{\mathrm{i}+1,2 \mathrm{j}} / 1 \leq \mathrm{i} \leq \mathrm{m}-1,1 \leq \mathrm{j} \leq 2^{\mathrm{i}}\right.$ \}.Then $|\mathrm{V}|=2^{\mathrm{m}+1}-1$ and $|\mathrm{E}|=2^{\mathrm{m}+1}-2$.

Consider the following two cases.

Case 1: $\mathrm{m} \geq 2$.

Define $\mathrm{f}: \mathrm{V} \rightarrow\left\{ \pm 1, \pm 2, \ldots, \pm\left(2^{\mathrm{m}+1}-1\right\}\right.$ as follows: $\mathrm{f}\left(\mathrm{x}_{0}\right)=1, \mathrm{f}\left(\mathrm{x}_{1,1}\right)=3, \mathrm{f}\left(\mathrm{x}_{1,2}\right)=-3, \mathrm{f}\left(\mathrm{x}_{2,1}\right)=-2, \mathrm{f}\left(\mathrm{x}_{2,2}\right)=-7$, $\mathrm{f}\left(\mathrm{x}_{2,3}\right)=2, \mathrm{f}\left(\mathrm{x}_{2,4}\right)=5$.

For $1 \leq \mathrm{i} \leq \mathrm{m}, 1 \leq \mathrm{j} \leq 2^{\mathrm{i}}$

Subcase 1.1: i is odd.

Example 2.5: In Figure 6, a pair sum labeling for the theta graph $\Theta\left(7^{[6]}\right)$ is exhibited.

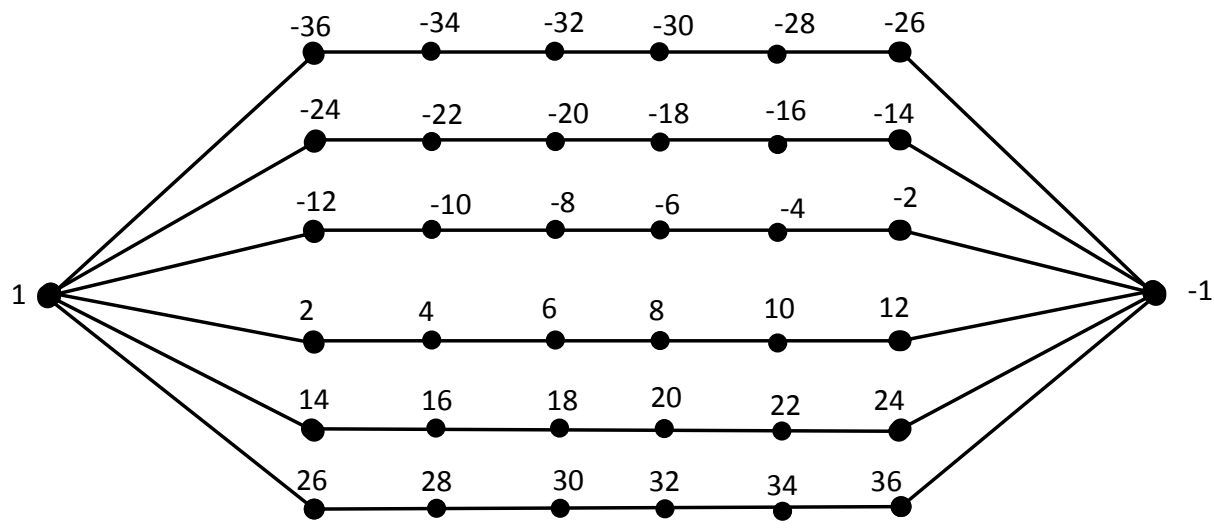

Figure 6: pair sum labeling for the theta graph $\Theta\left(7^{[6]}\right)$

Next we will prove that a complete binary tree is a pair sum graph. In a binary tree a vertex $v$ is said to be at level $d$, if $v$ is at a distance of $\mathrm{d}$ from the root. The number of vertices at levels $0,1,2, \ldots, \mathrm{m}$ in a m-level complete binary tree are $2^{0}, 2^{1}$ $, 2^{2}, \ldots, 2^{\mathrm{m}}$ respectively. Thus the total number of vertices in $\mathrm{m}$ level complete binary tree is $2^{\mathrm{m}+1}-1$.

Example 2.6: A 3-level complete binary tree is shown in Figure 7.

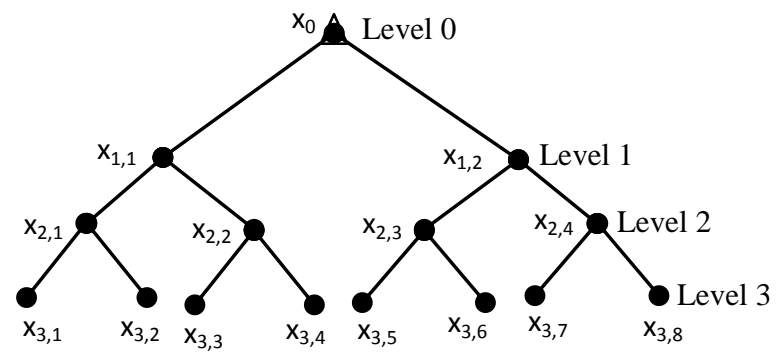

Figure 7 : 3-level complete binary tree

Theorem 2.7: For all $m \geq 1$, the m-level complete binary tree is a pair sum graph.

$$
\mathrm{f}\left(\mathrm{x}_{\mathrm{i}, \mathrm{j}}\right)= \begin{cases}-2^{\mathrm{i}}-j+1 ; & 1 \leq j \leq \frac{1}{2} 2^{i} \\ 2^{\mathrm{i}-1}+j-1 ; & \frac{1}{2} 2^{i}+1 \leq j \leq \frac{3}{4} 2^{i} \\ 2^{\mathrm{i}-1}+j+1 ; & \frac{3}{4} 2^{i}+1 \leq j \leq 2^{i}\end{cases}
$$

Subcase 1.2: i is even.

$$
\mathrm{f}\left(\mathrm{x}_{\mathrm{i}, \mathrm{j}}\right)= \begin{cases}-2^{\mathrm{i}}-j+1 & ; \quad 1 \leq j \leq \frac{1}{4} 2^{i} \\ -\left(2^{\mathrm{i}}+j+1\right) & ; \quad \frac{1}{4} 2^{i}+1 \leq j \leq \frac{1}{2} 2^{i} \\ 2^{\mathrm{i}-1}+j-1 & ; \quad \frac{1}{2} 2^{i}+1 \leq j \leq 2^{i}\end{cases}
$$

Then it is clearly observed that $\mathrm{f}$ is a pair sum labeling.

Case 2: Let $\mathrm{m}=1$. Then $\mathrm{V}=\left\{\mathrm{x}_{0}, \mathrm{x}_{1,1}, \mathrm{x}_{1,2}\right\}$ and $\mathrm{E}=\left\{\mathrm{x}_{0} \mathrm{x}_{1,1}\right.$, $\left.\mathrm{x}_{0} \mathrm{x}_{1,2}\right\}$. Define $\mathrm{f}: \mathrm{V} \rightarrow\{ \pm 1, \pm 2, \pm 3\}$ by $\mathrm{f}\left(\mathrm{x}_{0}\right)=-2, \mathrm{f}\left(\mathrm{x}_{1,1}\right)=$ $3, f\left(x_{1,2}\right)=1$. Clearly $f$ is a pair sum labeling.

Hence for all $m \geq 1$, the $m$-level complete binary tree admits a pair sum labeling.

Example 2.7: In Figure 8, pair sum labeling of fourlevel complete binary tree is illustrated. 


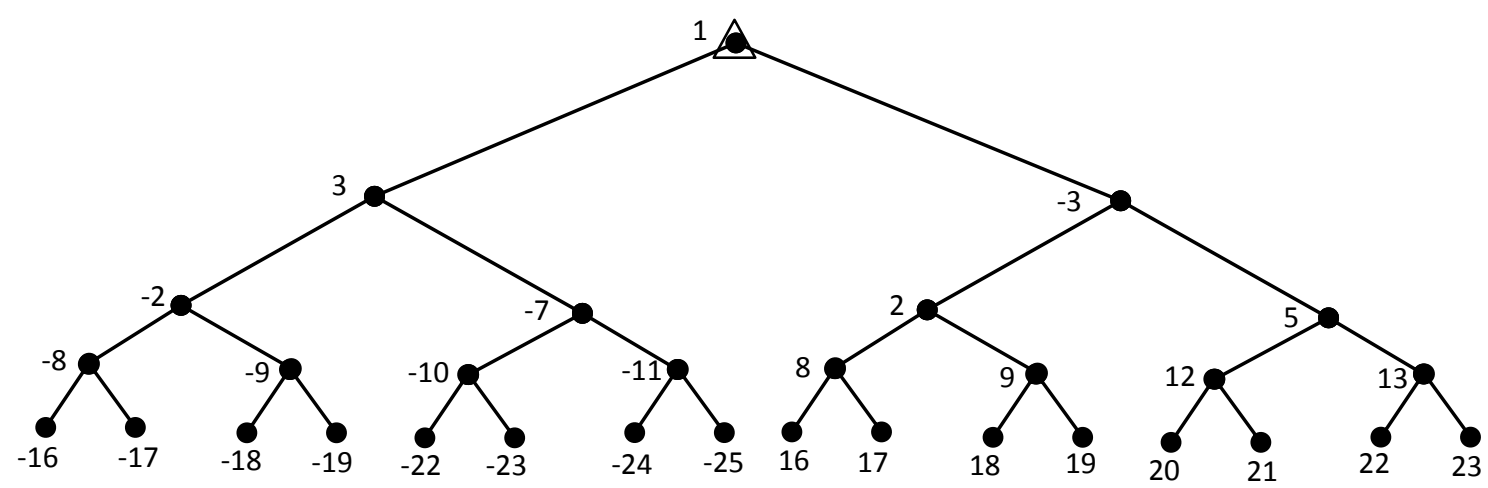

Figure 8: pair sum labeling of four level complete binary tree

\section{CONCLUSION}

In this paper we investigated some graphs like the coconut tree $\mathrm{CT}(\mathrm{m}, \mathrm{n})$, the $\mathrm{Y}$-tree $\mathrm{Yn}+1$, Jelly fish graph $\mathrm{J}(\mathrm{m}, \mathrm{n})$, the $(\mathrm{m}, 2)$-kite, $(\mathrm{m}, 1)$-kite, the theta graph $\Theta(\mathrm{l}[\mathrm{m}])$, for $\mathrm{m}$ even and complete binary tree are pair sum graphs. For the graphs like trees, $(\mathrm{m}, \mathrm{n})$-kites for all $\mathrm{n}>2$ this labeling can be verified.

\section{ACKNOWLEDGEMENTS}

Authors are thankful to the referee for the valuable comments which improve the standard of the paper.

\section{REFERENCES}

[1] J.A.Gallian, A dynamic survey of graph labeling, The Electronic Journal of Combinatorics, 14 (2009), DS6.

[2] F.Harary, "Graph Theory", Narosa Publishing House, New Delhi, (1998).

[3] R.Ponraj, J. V. X. Parthipan, "Pair Sum Labeling of Graphs", The Journal of Indian Academy of Mathematics, Vol. 32, No. 2, 2010, pp. 587-595.

[4] R.Ponraj, J. V. X. Parthipan and R. Kala, "Some Results on Pair Sum Labeling", International Journal of Mathematical Combinatorics, Vol. 4, 2010, pp. 5561.

[5] R.Ponraj, J. V. X. Parthipan and R. Kala, “A Note on Pair Sum Graphs", Journal of Scientific Research, Vol. 3, No. 2, 2011, pp. 321-329.

[6] R.Ponraj, J. V. X. Parthipan, Further Results on Pair Sum Labeling of Trees, Applied Mathematics, 2011, 2, 1270-1278.

[7] B. West, Introduction to Graph Theory, Prentice-Hall, India, 2001. 